\title{
Migration et tradition pragmatique en sociologie : une relation nécessaire?
}

\section{Pierre Tripier}

\section{(2) OpenEdition \\ 1 Journals}

\section{Édition électronique}

URL : https://journals.openedition.org/remi/1604

DOI : $10.4000 /$ remi. 1604

ISSN : $1777-5418$

Éditeur

Université de Poitiers

\section{Édition imprimée}

Date de publication : 12 décembre 2002

Pagination : 25-40

ISBN : 2-911627-32-6

ISSN : 0765-0752

\section{Référence électronique}

Pierre Tripier, « Migration et tradition pragmatique en sociologie : une relation nécessaire? », Revue européenne des migrations internationales [En ligne], vol. 18 - $n^{\circ} 3$ | 2002, mis en ligne le 09 juin 2006, consulté le 15 avril 2022. URL : http://journals.openedition.org/remi/1604 ; DOI : https://doi.org/ 10.4000/remi. 1604

Ce document a été généré automatiquement le 15 avril 2022.

(c) Université de Poitiers 


\title{
Migration et tradition pragmatique en sociologie : une relation nécessaire?
}

\author{
Pierre Tripier
}

1 Rompant avec une première époque de la sociologie américaine (Vidich et Lyman,1985), où l'état normal des comportements individuels et collectifs était l'adhésion sans réserve au christianisme réformé, l'esprit pragmatique est séculier et doit aller à la recherche des faits, sans s'embarrasser de leurs principes logiques :

«Aux débuts des années mil huit cent soixante-dix un noyau de jeunes gens de old Cambridge prirent l'habitude de se réunir régulièrement et se nommèrent, mi par ironie, mi par défi "Le club de métaphysique", car l'agnosticisme était alors fortement partagé et s'attaquait superbement à toute métaphysique. [...] Chauncey Wright, qui était alors considéré comme une célébrité en philosophie, ne manquait pas une seule de nos réunions. J'étais sur le point de l'appeler notre coryphée, mais il pourrait mieux être décrit comme notre maître en boxe, que nous particulièrement moi - affrontions en recevant de lui de sérieux horions. [...] Wright, James et moi étions des scientifiques, analysant les doctrines métaphysiques à partir de leur aspect scientifique plutôt qu'examinant leurs architectures idéelles. Le genre de notre pensée était très anglais. J'étais le seul à avoir été initié à la philosophie par la lecture de Kant, [...] mais même mes idées avaient l'accent anglais. [...] Nos échanges métaphysiques étaient confiés à des mots volants [...] aussi, lorsque le club devait se dissoudre, j'écrivis un petit essai dans lequel je compilais certaines des idées que j'avais défendues lors de nos rencontres, sous le titre de "pragmatisme". [...] Ce papier fut reçu avec une chaleur inattendue (par le public)» (Pierce, $1935: 1-157)$.

2 Parmi ces jeunes gens, l'un fera une très brillante carrière judiciaire puisqu'il achèvera son existence professionnelle à quatre-vingt onze ans en quittant la Cour Suprême des États-Unis dont il fut juge pendant 30 ans. Il s'agit d'oliver Wendell Holmes Jr., qui semble à bien des égards important pour notre propos puisqu'il entreprendra une des premières études pragmatiques sur le droit et, pour répondre à cette question établit ce qui sera un modèle pour l'étude des professions. Holmes s'est rendu célèbre jusqu'aujourd'hui, où il sert de référence dans les analyses sur l'évolution du droit aux 
États-Unis, par la phrase d'ouverture de son ouvrage The Common Law : «L'évolution de la loi n'a jamais été logique : elle est le fruit de l'expérience» (Maclouf, 2000). Il défend le point de vue suivant : beaucoup de commentateurs de la loi considèrent celleci comme dépendante de la volonté du souverain et reposant sur une logique qui lui est propre, la logique juridique. Mais il faut renverser cette proposition, car la loi dépend en définitive, dans la conception américaine, de ce que décident les juges. C'est donc une étude à la fois cognitive dans la mesure où interviennent leurs conceptions de la doctrine et de l'opportunité, de la situation dans laquelle ils jugent et s'est déroulé l'incident jugé ; et professionnelle, dans la mesure où la façon dont la loi sera dite, donc évoluera, est incompréhensible si on ignore comment se compose et se recrute le milieu même des juges .

3 Pour Holmes, «la loi est ce qui est accepté et renforcé par la cour de justice. Il ne s'agit pas de la volonté du souverain mais ce que les juges prétendent qu'il est (..) la seule question pour les avocats et procureurs est la suivante : comment le juge va agir? (Mills, 1964 : 110). Il nous paraît que dans cette démarche est annoncée une sociologie s'éloignant des types idéaux construits pour justifier l'existence, individuelle ou collective, d'une profession.

4 En effet nous quittons ici les impératifs déontiques pour des points de vue empiriquement plus faciles à vérifier et on marque bien, en même temps, la différence entre un monde de principes moraux et institutionnels et le monde effectif dans lequel se déroulent les activités humaines: si la corporation des juges dit qu'elle obéit à le volonté du souverain et à la logique juridique, le chercheur pragmatiste doit comprendre comment cette obéissance et cette logique sont mises en œuvre, en chemin il découvrira que le juge réinterprète ce rôle, au vu de son expérience et de sa lecture de la loi et pourra s'interroger sur les pourquoi et comment d'une rhétorique d'affirmation de son rôle qui en donne une version idéelle.

5 Cette insistance sur les pratiques va marquer la dernière polémique opposant les tenants de la tradition de Chicago et le courant structuro-fonctionnaliste, incarné avant tout par Parsons. En effet, les premiers travaux de cet auteur, jusque et y compris sa " somme » sur le système social (Parsons, 1951) sont pleins de polémiques sous-jacentes visant à imposer une sociologie plus déductive fondée sur ce que les économistes appellent une "réalité stylisée ", c'est-à-dire un type idéal, une version épurée des faits collectifs, des processus et des dynamiques historiques. Le fondement de ces discussions se trouve dans un affrontement avec les théories de Mead et leur «noyau dur », celui de l'« atome de socialité ».

6 On se souvient en effet que l'approche de Mead établit une théorie générale de l'ordre social qui, loin de traiter la société comme une structure, une totalité, part du plus petit segment social possible, la relation entre deux individus, selon un raisonnement qui se résume ainsi :

7 - l'« atome de sociabilité », premier lien social, est schématisé par une "conversation de gestes » dans laquelle un individu (homme ou animal) fait un mouvement vers un second individu, que celui-ci interprète, dont il anticipe la suite et auquel il répond.

8 - Cette conversation muette suppose que le système nerveux central ait enregistré les informations permettant l'interprétation et l'anticipation du geste d'autrui.

9 - Aucune information sur le monde extérieur n'est disponible chez le nouveau-né. La relation avec autrui constitue son stock d'informations, construit sa façon d'être au monde, de vivre en communauté, d'agir. 
10 - C'est sa capacité à jouer avec les règles de vie en commun, d'inventer des réponses à une situation, d'être à la fois "moi ", (répondre aux injonctions de l'« autrui généralisé » qu'est la communauté dans laquelle il vit), et à la fois « je » (construire, à partir de ces réponses routinières, des actions inédites) qui distingue l'homme de l'animal. Les conversations entre « je » et « moi » forment le « soi », c'est-à-dire l'être humain tel qu'il est effectivement.

«Le soi est moins une substance qu'un processus par lequel la conversation par gestes a été intériorisée par l'organisme. Ce processus n'existe pas par lui-même ; il est simplement une phase de toute l'organisation dont dépend l'individu. [...] Le processus qui lie un organisme aux autres dans les interactions qui se déroulent constitue le soi, dans la mesure où ce processus est transporté dans la conduite de l'individu par la conversation du "je" et du "moi". [...] (Chez l'homme), le processus social, avec ses différentes implications, est réellement assumé dans l'expérience de l'individu ; ainsi, ce qui a lieu se réalise avec plus d'efficacité (que chez l'animal), car, en un sens, l'individu l'a déjà répété.

La nature même de la conversation par gestes demande que l'attitude de l'autre soit modifiée par celle du premier qui réagit au stimulus de cet autre. Dans la conversation par gestes entre animaux, on peut noter ce jeu d'attitudes car l'individu non seulement s'adapte à celle des autres, mais encore les modifie. La réaction d'un individu, dans une telle conversation par gestes, transforme continuellement, à quelque degré, le processus social lui-même. C'est cette modification du processus qui est du plus grand intérêt dans l'expérience de l'individu. Il adopte l'attitude d'autrui envers son propre stimulus, et, en l'adoptant, il remarque qu'il modifie sa réaction, laquelle, à son tour, conduit à de nouveaux changements [...].

Aucun individu ne peut réorganiser toute la société; mais l'homme affecte continuellement la société par sa propre attitude, parce qu'il prend l'attitude du groupe envers lui et y réagit » (Mead, 1963 : 152-153).

11 Une fois finies les années d'apprentissage, les conversations entre «moi » et «je » s'estompent. Le « soi » achevé s'interprète lui-même en tant que « je ", interprète son action comme simple invention ou réaction à la situation, en gommant tout ce qui, dans cette action, est redevable des interactions avec autrui, à cet immense stock de connaissances, règles, normes, manières de voir, de ressentir et d'agir qui lui ont été, partiellement, transmises par son entourage.

12 Ce mouvement de "naturalisation », $d$ ' " enfouissement » des règles de conduite intériorisées préside aux interprétations individualistes de l'action, expliquant pourquoi l'ordre social apparaît sous la forme de relations individuelles ; ordre existant avant les individus, même si ceux-ci, grâce à leur créativité, le bouleversent.

13 La théorie de Mead explique, à partir de cet atome social, la permanence et le changement dans une société; la permanence par le renforcement que chaque communauté donne à ses actions répétitives en les instituant; le changement car chaque communauté (qu'il appelle aussi autrui généralisé) se transforme (1) dans l'interaction avec les autres communautés (2) grâce à la créativité de ses membres mais aussi aux relations qu'ils entretiennent en se rencontrant.

14 Considérant que chacun est le fruit des interactions qu'il a vécues, au moins pendant les années d'apprentissage, les chercheurs de Chicago auront tendance à transposer les contraintes que Darwin avait jugées majeures dans l'analyse sociologique élaborée par Mead. Les ressources territoriales, les phénomènes de nombre, d'invasion et de rejet deviendront pour eux des variables fortes dans l'explication des phénomènes sociaux. 
15 Mais la qualité des groupes compte aussi; une qualité qui n'est pas seulement héréditaire puisqu'elle se construit aussi dans de multiples interactions. Comme l'avait pressenti Darwin, celles-ci forgent la particularité des individus, des groupes et des communautés. Aussi jouent-elles, pour les groupements humains, le rôle du hasard; elles permettent à ces groupements de se doter de qualités potentielles, utilisées, peutêtre plus tard, si l'environnement change, si un danger, une invasion, un conflit, les menacent.

Les interactions avec autrui ne sont pas seulement d'ordre conflictuel, menaçant. Pour Park et ses disciples, le conflit n'est qu'un moment dans la relation entre les groupes et entre les personnes, car peuvent survenir d'autres relations, de transaction, d'accommodement réciproque, ou cette assimilation, cette disparition des différences, cette culture nouvelle née de l'interaction, que le «Melting Pot » américain illustre si bien.

Le modèle de l'atome de sociabilité joue son rôle ici dans le contact avec des voisinages, des métiers, des pédagogies différents. L'image d'une communauté unique d'un « autrui généralisé » monolithique laisse la place à la présence de plusieurs communautés, de plusieurs milieux et d'une multitude de "mondes». On ne peut pas expliquer la réaction d'une personne devant une autre personne en termes généraux, il faut se référer à son parcours antérieur, à son expérience. Elle lui permettra de définir la situation dans laquelle elle se trouve, et dans laquelle se trouve son vis-à-vis. Cette définition de la situation (de soi et de l'autre), organise les relations entre les personnes mais aussi entre les groupes, vicinaux, ethniques ou professionnels.

La définition de soi et des autres permet de comprendre la façon dont chacun va agir et réagir dans une situation de co-présence et comment vont se créer ainsi de la coordination et de la désorganisation provenant de la compréhension, du malentendu ou de l'incompréhension de la façon dont l'autre définit la situation.

Ceci explique aussi que se développent, notamment dans les villes, des mécanismes d'apprentissage qui empiètent très loin sur l'âge adulte. On doit ainsi voir dans l'insistance que mettent les chercheurs de l'École de Chicago à étudier les immigrants non seulement un reflet de la situation contemporaine du peuplement des États-Unis, mais aussi une excellente illustration de leur théorie. Le migrant, qui est en période d'apprentissage plus longtemps que d'autres, permet d'examiner, chez lui et grâce à lui, de façon presque expérimentale, comment se transforme une personnalité, déjà adulte, au contact d'autres communautés.

Donc les individus, les groupes font preuve de créativité, tout en se modifiant au contact des autres. Ils se dotent, sans le savoir peut-être, de traits culturels qui joueront leur rôle lorsqu'il faudra affronter le changement ou qui les empêcheront de le faire.

On rejoint ici l'autre versant de la sociologie pragmatique : donner de l'importance au temps et à son caractère irréversible. Transposée à l'échelle humaine cette préoccupation temporelle permet de mieux situer les analyses interactionnistes : si ce sont les interactions et la créativité des individus et des groupes qui expliquent leur survie ou la position qu'ils occupent, dans l'espace géographique ou social, il ne saurait y avoir d'explication sociologique d'une situation, d'un événement, sans l'élucidation des interactions qui l'ont produit. L'analyse sociologique est historique.

23 C'est contre ce point de vue que va se révolter Parsons. En effet, cette sociologie meadienne interdit tout propos généralisateur puisqu'elle sous-estime très largement 
deux des ressorts de l'universalisme sociologique conçu par lui : le langage et les institutions.

Dans ses exposés, Mead mélange allègrement des exemples animaux et des exemples humains; il montre, contre bien des tendances de la biologie de son époque que certaines parties de ce que l'on appelle l'instinct animal est en fait un apprentissage, faits désormais reconnus grâce aux énormes progrès de l'éthologie. Mais ceci constitue pour Parsons un crime de lèse majesté, aussi propose-t-il de substituer au modèle de l'atome de sociabilité, celui de la « double contingence» :

«Toute analyse empirique de l'action suppose la prise en compte des capacités biologiques. [...] même les orientations de l'action les plus élémentaires du niveau animal suppose un début de symbolisation [...]. Au niveau humain un pas est fait de l'action orientée par les signes à la vraie symbolisation. [...] Dans les situations classiques d'apprentissage animal, celui-ci est devant des alternatives entre lesquelles il fait une sélection et développe des attentes que certaines indications viennent confirmer. [...] Quels que soient les origines et les développements des systèmes de symboles il est certain que l'élaboration des systèmes élevés de l'action humaine ne sont pas possibles sans un système symbolique relativement stable. [...] Ce système symbolique partagé qui fonctionne en interaction est ce que nous appelons une tradition culturelle » (Parsons, 1951 : 9-11)

À partir de ces prémices, Parsons va proposer d'analyser l'interaction dans une relation entre rôle et status, eux-mêmes organisés par un système culturel, lequel se maintient grâce à l'action des institutions. Il n'est pas étonnant que dans ce schéma le système social devienne complètement synchrone, il n'a aucune dynamique propre, celle-ci lui vient de l'extérieur, dans un mouvement linéaire d'évolution. Mais l'insistance sur les rôles et status, sur les institutions et la culture conduit à surestimer les règles formelles, les présupposés et injonctions culturelles. Dans son illusion de pouvoir tenir un discours exhaustif sur la société et son évolution, le sociologue structurofonctionnaliste durcit les réalités tout en les appauvrissant, en les réduisant à leur idéal ou à leur stéréotype.

A contrario, le courant de l'École de Chicago s'inscrit dans une démarche synthétisée par cet extrait de Simmel :

«Le moment présent dans l'évolution de la science sociale semble correspondre à l'état où se trouvèrent les sciences de la vie organique lors des commencements des recherches microscopiques. Jusqu'alors les recherches avaient été limitées aux organes corporels se distinguant nettement et ayant une certaine grandeur, organes dont la fonction et les formes présentaient des différences accusées. À ce moment-là pour la première fois se révéla la liaison qui existe entre le processus vital et les plus petits des éléments qui leur servent de base, les cellules; et en même temps se révéla l'identité existant entre celui-ci et les actions innombrables et continues qui se produisent entre ces éléments.

Ce n'est qu'en approfondissant la façon dont les cellules s'attirent ou se repoussent, se détruisent les unes les autres ou produisent entre elles des réactions chimiques que l'on peut graduellement comprendre comment un corps prend sa forme, la conserve ou l'altère. Une science ne tenant compte que du cœur et des poumons, de l'estomac et des reins, du cerveau et des organes moteurs, grands organes dans lesquels les acteurs essentiels de la vie et leurs réciprocités d'action se sont réunis en des formes distinctes et des fonctions macroscopiques, n'aurait jamais pu concevoir l'ensemble de la vie [...]. Aujourd'hui la science sociale en est généralement encore à cette période où elle observe seulement les plus grandes formes sociales, celles qui sautent aux yeux, et c'est en se basant sur celles-ci qu'elle veut édifier dans son ensemble la connaissance de la vie sociale... » (Simmel, 1981 : 223-224). 
Ainsi, il existe dans la vie collective des données faibles ou molles et des données fortes ou dures. Toutes les données, qui sont produites en relation avec des phénomènes institués, légalisés, juridicisés, qui se rapprochent davantage des textes que des pratiques peuvent être considérées comme dures. Socialement situées, elles donnent lieu à des comptages, des mesures, on en suit les proportions. On en repère statistiquement l'évolution. Dans l'état collectif qui précède une action ou qui forme son contexte, les institutions, les lois, les mœurs et les coutumes sont considérés comme ayant un poids spécifique de grande importance. Nous considérons qu'ils pèsent autant que les contraintes comme la rareté des ressources ou le nombre de concurrents. Cependant les résultats collectifs de recherche ont montré, que c'est en étudiant les processus mis en place par ces lois, ces règlements et ces institutions que l'on comprenait leur fonctionnement, plutôt qu'en les interrogeant directement. Ce qu'apporte l'approche pragmatiste n'est pas une négation des données fortes mais de leur relative importance. Ce point avait déjà été développé par Darwin, quand il avait remarqué que ce ne sont pas les caractéristiques les plus visibles d'une famille qui distinguent les espèces qui la composent. En somme que les traits différenciateurs sont souvent des données faibles ou muettes.

Il en serait de même dans l'écologie humaine, les faits les plus visibles, ceux qui sont codifiés dans les lois et règlements, qui auraient les honneurs des philosophies politiques et, de ce fait, tiennent le haut de la scène, ne seraient pas les plus importants dans le mouvement historique étudié par les approches pragmatistes. Un prodigieux exemple de ce qui est ici avancé a été développé par J.P. Briand et J.M. Chapoulie dans leur ouvrage sur le primaire supérieur. Alors que, pendant soixante-dix ans, une discussion enflammée porte sur la scolarisation des garçons, c'est l'éducation des filles, leur entrée dans le primaire supérieur, qui sera grosse de la modernisation ultérieure de la société et de la place relative des familles. À l'époque, ce processus est muet, un structuro-fonctionnaliste ne l'aurait jamais perçu (Briand et Chapoulie, 1994).

Dans cette démarche pragmatique, la découverte est valorisée, or celle-ci consiste avant tout à faire parler des données muettes, à considérer symétriquement les données faibles et les données fortes.

\section{Le sens de la dynamique historique}

Comment rendre compte de façon pragmatiste du mouvement historique sans tomber dans d'énormes banalités, sans faire penser que le destin des peuples consiste à suivre la voie tracée par les plus riches d'entre eux ou attendre l'épuisement du concept d'une époque pour voir apparaître la suivante, ou le passage d'un mode de production à un autre ? Pour mieux illustrer ce qui se jouerait dans la dynamique historique, rappelons que, dans le sens de l'avancement des sciences évoqué par Simmel, celles de la nature ont progressé en s'intéressant aux individualités. E. Mayr nous le rappelle : en biologie " on a très rarement affaire à des classes d'identité, presque toujours on doit étudier des populations consistant en individus, chacun unique à sa façon. Cela est vrai à tous les niveaux hiérarchiques: de la cellule aux éco-systèmes de nombreux phénomènes biologiques sont caractérisés par des variances élevées. La vitesse d'évolution ou les taux de spéciation differrent d'un cas à l'autre par trois à cinq ordres de grandeur, degrés rarement atteints par les objets du monde physique... » (Mayr, 1989 : 394) et que ne pourrait-on dire de l'espèce humaine ? 
31 L'approche pragmatique du devenir historique sera donc individuelle : c'est en suivant les parcours des personnes, la façon dont ils avancent dans leur chemin vocationnel, les coïncidences de leur cycle de vie avec les événements de la vie privée et publique, professionnelle et familiale que l'on va le mieux parler des mouvements historiques.

Dans les itinéraires biographiques apparaissent les efforts infructueux et ceux couronnés de succès, le roman des vaincus et celui des vainqueurs, la succession des familles dans des places et leurs départs dans d'autres status, les mouvements des personnes dans les villes et leur passage de quartier en quartier. Parce qu'on étudie des individualités en mouvement, on résout le problème lancinant du changement social. Comme dans le «De Motu » de Galilée, le changement, le mouvement n'est pas conçu comme une exception mais bien l'état normal.

On saisit la dynamique de l'histoire en retraçant les sentiers parcourus par les individualités que l'on étudie. Le paysan polonais sera ainsi pisté par Thomas et Znaniecki, de son village où il vit confiné par le respect des notables et l'obéissance à l'autorité du prêtre, à la ville polonaise où, libéré de ces liens, il s'enivre de liberté et entre dans une période de désorganisation sociale, pour ensuite réorganiser ses relations aux autres dans les quartiers polonais de Chicago, reconstruisant alors, quelquefois de façon mythique, les éléments de solidarité qu'il avait connus dans son enfance.

Sous l'influente pensée de Dewey, ce mouvement n'est pas seulement pensé comme un parcours sur une sinusoïde morale, il est aussi vu comme une évolution cognitive : "Chaque organisme distinct, structure ou formation, chaque groupement de cellules ou d'éléments, doit être étudié comme un instrument d'ajustement ou d'adaptation à une situation environnante particulière. Son sens, son caractère, sa valeur, sont connus quand et seulement quand on détecte la nature de l'arrangement qui permet de survivre dans les conditions particulières d'une situation spécifique » (1938: 81).

35 Ce cognitivisme adaptatif semble guider l'approche des sociologues pragmatiques, ainsi Thomas et sa conception la plus centrale, celle de définition de la situation.

De tous les concepts de Thomas, c'est celui de « définition de la situation » qui a connu la plus grande postérité, probablement parce qu'il s'est trouvé relayé, dans les générations successives de sociologues, par R.K. Merton qui l'utilise dans un texte ayant trouvé grâce aussi bien aux yeux des structuro-fonctionnalistes que des partisans de l'individualisme méthodologique. Ce texte, traitant de la prédiction créatrice, commence par ces mots: "Dans une série de travaux auxquels les universitaires sont à peu près les seuls à se référer, le doyen des sociologues américains W.I. Thomas, a formulé un théorème essentiel pour les sciences sociales: "Quand les hommes considèrent certaines situations comme réelles, elles sont réelles dans leurs conséquences". Si ce théorème et ses incidences étaient mieux connus, moins rares seraient ceux qui comprennent le fonctionnement de notre société » (Merton, 1987 : 169).

Cependant les familiers des travaux de Thomas ne manqueront pas de remarquer le caractère réducteur de la version que Merton donne de ce théorème, réduction qui a peut-être assuré son succès. En effet, pour l'auteur de The Unadjusted Girl, la définition de la situation ne se fait pas à l'initiative d'une personne, cette définition est à proprement parler réciproque. Quelqu'un définit une situation et agit en conséquence, mais cette définition peut se heurter à une contradiction, faite par autrui, par son entourage ou encore par les mœurs ou les lois. Elle encourt alors le risque d'être 
démentie ou même sanctionnée, non seulement à cause des résultats de son action mais aussi en vertu des principes qu'elle a mis en œuvre, avec la bonne foi de l'ignorance, ou la perversité de la rationalisation.

«L'individu ne trouve jamais passivement de situations toutes faites similaires à des situations passées; il lui faut définir consciemment chaque situation comme étant similaires à certaines situations passées s'il veut lui appliquer la même solution [...]. Et c'est précisément ce que la société attend lorsqu'elle exige de lui une organisation de vie stable. [...] L'uniformité de comportement est une uniformité de règles observées consciemment » (Thomas et Znaniecki, $1998: 59$ ). chercheur, lui apportant une information inédite, viendrait défaire une théorie trop bien agencée. Ses chercheurs étaient des gens d'expérience, ancien camionneur, ancien chef de bande, ancien travailleur social spécialisé dans les gangs ; il recevait également des élèves frais émoulus de Chicago et des marginaux, avec une préférence pour ces derniers.

42 E. Burgess, qui partageait avec lui la direction du Département, était lié aux milieux huppés de Chicago, dont les dons avaient permis la création de la première revue de sociologie des USA, pendant longtemps la seule qui servit de moyen d'expression à la société savante l'American Sociological Association. Ces dons permettaient de posséder une des meilleures bibliothèques où les sciences de la vie étaient aussi bien représentées que les sciences sociales et humaines, de publier aussi les thèses des étudiants et les travaux de chercheurs, de remarquables monographies qui restent encore comme des classiques. Quelques-unes sont traduites en français, comme «Le Ghetto » de Wirth ou « Le Hobo » d'Anderson.

La force d'un département dans les circonstances de Chicago était bien dans le mélange des traditions, la gradation de l'initiation, la proximité de la recherche vivante, la possibilité de la diffuser très vite, mais aussi dans l'existence d'une bibliothèque avec en stock toutes les traditions scientifiques. 
Est-ce à dire que Park et Burgess n'apportèrent aucune contribution conceptuelle à la sociologie ? Il faut leur reconnaître à la fois des apports à la sociologie de la ville et de ses habitants et à la sociologie des relations intercommunautaires.

5 La place de cette dernière se comprend aisément dans le paysage intellectuel si l'on tient compte du moment fondateur de la nation américaine : l'arrivée sur le Mayflower d'une communauté puritaine, unie, égalitaire et débarquant dans un milieu étranger après avoir quitté une Angleterre hostile.

6 Le fait que leur nation soit issue d'une communauté dissidente imprègne jusqu'aujourd'hui les mentalités des habitants des États-Unis en leur faisant voir la ou les sociétés comme un conglomérat de communautés différentes. Cela était peut-être encore plus vrai au début du siècle quand des vagues d'immigrants, venant d'abord des pays protestants européens (Allemagne, Suède), puis des pays catholiques ou orthodoxes (Irlandais, Italiens, Polonais, Russes, Serbes, etc.) arrivaient par dizaines de milliers. Observant comment se comportent les anciens habitants vis-à-vis des plus nouveaux, les sociologues de Chicago remarquaient des régularités, dont ils pouvaient d'ailleurs suivre la réalité sur les plans mêmes de leur ville en y reportant les mouvements de population. Ils ne pouvaient aucunement s'engouffrer dans l'opposition métaphysique qui contrastait dans les pays européens communauté et société et attribuait à la première tous les charmes de l'interconnaissance et de l'équilibre social, puisqu'ils voyaient arriver par bateaux entiers des personnes dont le seul rêve était de fuir à tout jamais leur village natal et se fondre dans les relations choisies qu'offre la ville.

Dans ce monde mouvant, soumis à un incessant flux de population, des dynamiques irréversibles et structurelles se mettent en route qui affectent les relations des personnes cohabitant sur les mêmes territoires ou sur des espaces voisins.

B Ces processus d'interaction peuvent être plus ou moins antagonistes. Reprenant une distinction de Simmel, Park et Burgess distinguent quatre concepts qui les caractérisent : la compétition, le conflit, l'accommodement et l'assimilation.

\section{Compétition, conflit, accommodement, assimilation}

49 Si l'interaction est la relation structurante la plus répandue entre les humains, la compétition apparaît comme sa forme la plus générale. Il faut entendre par là une façon d'être dans une société aux ressources rares et aux actions multiples. Il y a rivalité pour s'approprier les premières et pour utiliser d'autres êtres humains pour accomplir les secondes, mais cette rivalité peut être impersonnelle, s'imposer entre des personnes qui s'ignorent, qui ne se rencontreront jamais. La compétition est l'interaction sans contact, sans intercommunication.

«La compétition tend invariablement à créer un ordre collectif impersonnel dans lequel chaque individu, étant libre de poursuivre ses propres fins, et, dans une certaine mesure, étant poussé dans cette poursuite, transforme autrui en moyen pour atteindre ses buts [...]. Ce caractère externe des relations entre humains est un aspect fondamental de la vie en société [...]. La société est faite d'individus spatialement séparés, distribués territorialement et capables de se mouvoir par eux-mêmes. Cet accès à la locomotion indépendante est la base et le symbole de toutes les autres formes d'autonomie. La liberté est avant tout liberté de se mouvoir et l'individualité est inconcevable si elle est privée de la capacité et de l'opportunité d'acquérir une expérience individuelle grâce à la conduite d'actions autonomes. [...] 
La compétition crée l'ordre social et écologique. Elle détermine la distribution territoriale et professionnelle des individus. Elle produit la division du travail et l'organisation de l'interdépendance des individus et des groupes» (R.E. Park et E. Burgess, 1970 : 187-189).

Si la compétition apparaît aux deux auteurs de l'École de Chicago comme une sorte de socle commun à toute humanité, ne supposant pas cependant des interactions de face à face qui puissent les modifier, il n'en est pas de même des trois autres classes de relation entre personnes: le conflit, l'accommodement et l'assimilation. Ces trois figures de l'intercommunication se trouvent dans un continuum d'apaisement, de pacification des relations. Mais cela ne veut pas dire, aux yeux de leurs auteurs, qu'il s'agisse d'une évolution irréversible. Même si l'histoire naturelle de certaines communautés ethniques aux États-Unis montre le passage du conflit à l'assimilation, il faut se garder de faire de cette conjonction historique une loi de développement, comme le montre, à l'époque de Park et Burgess, le sort de toutes les communautés qui n'ont pas seulement des dissemblances linguistiques ou culturelles avec la majorité de la population ou son élite mais aussi des différences de pigmentation de l'épiderme. "Les contacts sociaux ne provoquent des relations conflictuelles nulle part de façon si aiguë que dans les rapports inter-raciaux, surtout quand les relations raciales sont renforcées par des différences de couleur. Nulle part non plus [...] les réponses aux contacts sociaux ne sont aussi évidentes et, dans le même temps aussi difficiles à définir et analyser » (R.E. Park et E. Burgess, $1970: 240$, Traduction : P.T.).

51 Dans une société, le conflit est rarement ouvert et il enrôle rarement des populations importantes numériquement. Il faut donc entendre le conflit dans un sens local et montant rarement aux extrêmes. Même si le conflit est réduit, il garde le caractère structurant que lui avait reconnu Simmel. Park et Burgess articulent ce concept à celui de définition de la situation : le conflit signifierait à chacun de quels droits il dispose, de quelle latitude il jouit, qui contrôle le territoire sur lequel il se trouve.

La lutte des classes, de Marx et de ses disciples, apparaît alors comme une forme particulière d'un conflit territorial, où la définition de la situation par le salarié, notamment les droits qu'il réclame en échange de son travail peuvent lui être contestés de façon ferme, voire brutale, par son employeur ou par L'État.

Les accommodements sont ces formes sociales qui permettent d'anticiper les dissensions et d'éviter les situations de conflit ouvert. Les accommodements sont organisés par les rites de salutation, les règles de préséance, l'intériorisation de la présence de l'autre. La situation d'accommodement est similaire à celle de l'adaptation écologique des biologistes, une co-présence d'espèces différentes leur permettant de survivre en équilibre. Mais son origine est humaine, elle s'organise sur un fond de conventions, mœurs, règles et lois qui permettent à des personnes habitées par des conceptions différentes d'occuper le même espace ou de faire appel à un juge pour qu'il les départage :

«L'accommodement est l'issue naturelle d'un conflit. Dans l'accommodement l'antagonisme entre des éléments hostiles se trouve, pour un certain temps, régulé. Le conflit, qu'il prenne la forme d'une guerre, d'une grève ou de simples échanges d'insinuations aussi polies que dévalorisantes, finit toujours, immanquablement, dans un nouvel ordre social ou accommodement. Cet accommodement s'obtient par la modification du statut réciproque des participants au conflit» (R.E. Park et E. Burgess, 1970 : 305). 
l'existence d'une compréhension mutuelle. Les conflits s'apaisent puisque la racine de leur existence a disparu. Désormais devenus américains, les migrants éprouvent un patriotisme encore plus brûlant que les anciens habitants et la nouvelle génération fonde ses différences sur d'autres critères que l'ancienne appartenance nationale. Park et Burgess vont étendre à d'autres situations, plus générales, le contact provoquant l'assimilation :

«L'assimilation est un processus de fusion, d'interpénétration dans lequel les personnes et les groupes font leur les souvenirs, les sentiments et les attitudes d'autres personnes ou groupes. Comme ils partagent les mêmes expériences et la même histoire ils se trouvent unis par de mêmes repères culturels [...]. Dans l'accommodement les changements d'attitude peuvent être brusques et revêtir une forme révolutionnaire, comme on peut le constater historiquement en examinant les conversions. Par contre les modifications d'attitude dans le processus de conversion ne sont pas seulement graduelles, mais peu perceptibles dans le temps court, alors qu'elles apparaissent très importantes quand on observe une période de temps suffisamment longue. [...] Le procès d'assimilation est typiquement inconscient: une personne peut facilement être incorporée dans le mode de vie d'un nouveau groupe sans y prêter attention ni pouvoir reconstituer l'enchaînement d'événements qui l'y ont conduit » (R.E. Park et E. Burgess, 1970 : 360-361).

\section{Conclusion}

Sans avoir pu l'approfondir davantage, on voit bien comment certains traits de la philosophie pragmatiste, telle qu'elle avait été élaborée par le Club de Métaphysique de Harvard imprègnent l'approche interactionniste.

Si le monde est fait à partir d'interactions, organisées à partir des différentes définitions de la situation et des différentes réactions qu'elles suscitent, le passé est toujours présent, avec ses légendes, ses mythes, son histoire reconstruite, ses normes et ses prescriptions, mais la créativité individuelle, de nouvelles rencontres, bouleversent à tout moment l'ordre des choses. Désormais, si le passé est présent mais aussi construit par les définitions réciproques de la situation (dans lesquelles la projection sur l'avenir probable de soi et de ses vis-à-vis joue un rôle très important), toute théorie générale de la société devient alors un effort inutile.

Pour cette approche des collectifs humains, la seule certitude est que l'ordre social est fondé sur des interactions, son aspect étant à chaque fois particulier. S'il en résulte des invariants, ce sont ceux que fournissent les sciences du vivant. Seule la recherche de terrain, le stock de connaissances amassées dans des protocoles rigoureux, peut permettre des généralisations circonspectes d'une réalité aussi susceptible de changements : leur auteur n'est jamais à l'abri d'une interaction qui, lui montrant une exception, le conduirait à abandonner son modèle d'explication. 
59 Aussi, dans cette perspective théorique, chercher à devenir un maître à penser, à dire la vérité sur le monde, à faire une théorie générale sur la société, est une entreprise trompeuse. Seule l'action, comme l'affrontement dans l'art de la guerre, condense, à un moment donné, dans un endroit donné, la réalité des choses, et son explication exhaustive suppose la maitrise de tant de paramètres qu'elle en devient impossible.

Les interactionnistes ne nient pas les autres paradigmes; ils se contentent de les relativiser, de prendre avec eux une distance telle que la vision d'une bonne société disparaît. Ils ne nient ni les violences du marché, ni celles de l'exécutif tout-puissant. Ils participent aux mouvements visant une meilleure égalité, comme en témoignent les biographies de Thomas et de Park, mais la vigilance du savant doit le prémunir de substantifier les solutions proposées comme remède, dont sa propre transformation en homme politique rationnel.

61 Comme chacun développe l'art de se présenter à autrui, surtout dans les villes où de multiples communautés coexistent et où les individus voyagent entre plusieurs, la présentation de soi devant le sociologue fait partie de ces multiples façons d'être avec autrui, de construire son discours dans le dialogue, en guettant chez autrui des réactions favorables, de sentir l'argument légitime, l'argument séducteur. La distance que les interactionnistes prennent vis-à-vis du discours que les professions, les communautés ethniques ou locales, tiennent sur elles-mêmes a pour base ce paradigme et se confond avec la logique dialogique.

On comprend mieux pourquoi les données longitudinales, dont l'histoire de vie, jouissent d'un privilège épistémologique dans cette configuration scientifique: l'histoire de vie permet de reconstituer les interactions qui, aux yeux d'une personne, ont compté dans son existence. L'histoire de vie met en scène des groupes plus ou moins amples, en interaction, qui ont forgé une partie du destin des personnes que l'on interroge. On peut, en les contrôlant par d'autres données longitudinales, saisir les multiples ramifications qui relèvent de leurs contacts privés et publics : la genèse d'une action, d'une conviction, d'une vision du monde. L'explication historique mêle alors le subjectif et l'objectif dans une analyse déictique : elle montre la réalité du doigt.

\section{BIBLIOGRAPHIE}

ANDERSON Nels (1993) Le Hobo, sociologie du sans abri, trad F., Paris, Nathan, 305 p.

BRIAND Jean-Pierre et CHAPOULIE Jean-Michel (1994) Les Collèges du Peuple : l'enseignement primaire supérieur et le développement de la scolarisation prolongée sous la Troisième République, Paris, INRP, édition du CNRS et de l'École Normale Supérieure de Fontenay-Saint Cloud.

BULMER Martin (1984) The Chicago School of Sociology, Chicago U.P.

DEWEY John (1938) Logique. La théorie de l'enquête, Paris, PUF. 
MACLOUF Pierre (2000) Crise de la « common law » et « nouvelle régulation juridique ", à propos de Mary Ann Glendon et du débat sur la transformation du droit aux États-Unis, Archives de Philosophie du Droit, 44, pp. 333-357.

MAYR Ernst (1989) Histoire de la Biologie : Diversité, évolution et hérédité, trad. F., Paris, Fayard.

MEAD George Herbert (1963) L'Esprit, le soi, la société, trad. F. Paris PUF.

MERTON Robert King (1987) La Prédiction créatrice in Élements de théorie et de méthode sociologiques, Brionne, Montfort.

MILLS C. Wright (1964) Sociology and Pragmatism : The Higher Learning in America, Londres, N.Y. Galaxy Books.

PARK Robert Ezra et W. BURGESS Ernest (1970) Introduction to the Science of Sociology, Chicago U.P. PARSONS Talcott (1951) The Social System, N.Y., Free Press.

PIERCE Charles (Santiago) Sanders (1935) What Pragmatism is, in Collected Papers Cambridge (Mass.) Belknap Press, 5 Tomes.

SIMMEL Georg (1981) Essai sur la sociologie des sens, in Sociologie et épistémologie, Paris, PUF.

THOMAS William Isaac et ZNANIECKI Florian (1998) Le Paysan Polonais en Europe et en Amérique, 5 volumes dont le $\mathrm{N}^{\circ} 3$ (Récit de vie d'un migrant) est traduit en français, Paris, Nathan.

VIDICH Arthur J. et M. LYMAN Stanford (1985) American Sociology : Wordly Rejections of Religion and Their Directions, Yale U.P.

WIRTH Louis (1980) Le Ghetto, trad. F. Grenoble, PUG.

\section{RÉSUMÉS}

La sociologie pragmatique s'est formée aux États-Unis en voyant le fondement de la vie collective dans la constitution de la personnalité de chacun par l'interaction avec les autres. C'est à partir de ce noyau social que les règles, mœurs et institutions deviennent intelligibles. Cette approche s'éloigne de toute transcendance: elle privilégie les données faibles, qui proviennent des processus d'apprentissage et des comportements individuels. Pour cette sociologie, la situation de l'Amérique du Nord qui voit des bateaux entiers débarquer des paysans fuyant les communautés rurales européennes et s'adapter à un univers inconnu : la ville nord-américaine, constitue un vrai laboratoire des conduites humaines. On y trouve en germe une vision territoriale des relations à autrui se manifestant dans les concepts de définition de la situation, compétition, conflit, accommodement et assimilation. Ils forment la trame légère d'une sociologie compréhensive et vivante.

Is there a Necessary Relationship between Migrations and the Pragmatic Tradition of Sociology? -- North American pragmatic sociology assumes collective life as being the effect of relationship between selves who build themselves in the interaction with others. Rules, mores and institutions grow up from this first social knot. This conceptualization of society doesn't need any transcendence and makes use of weak data, like learning processes and behavior of individuals. US conjuncture at the beginning of Twentieth Century was a laboratory situation to study human behavior. When boats came full of European peasants fleeing their rural communities and adapting themselves to an unknown situation: north American cities. To understand this situation a territorial vision of the relationship between self and others is 
needed. This vision requires concepts like definition of the situation, competition, conflict, accommodation and assimilation. These concepts frame a living and comprehensive sociology.

¿ Es necesaria la relacion entre las migraciones y la tradicion pragmatica en sociologia ? -- Los fundamentos de la sociología pragmatica norte americana toman sus raíces en el principio según el cual la vida colectiva se funda ante todo sobre el encruce de las personalidades, quienes se construyen en la interacción con otros. Si se tiene en cuenta esta origen, las reglas, costumbres e instituciones parecen derivadas de este núcleo social primero. Esta manera de pensar la sociedad se separa de toda trascendencia y utiliza sobre todo datos frágiles, los cuales nacen en los procesos de aprendizaje y en las conductas individuales. Para esta sociología, la situación de los Estados Unidos a principios del siglo veinte, la llegada de buques enteros de campesinos europeos huyendo sus comunidades rurales y adaptándose a la ciudad norte americana, un universo desconocido de ellos, constituye un verdadero laboratorio para estudiar las maneras de ser humanas. Permite el nacimiento de una visión territorial de las relaciones al otro, que produce los conceptos de definición de su situación, de competición, conflicto, acomodación y asimilación. Estos conceptos construyen el marco de una sociología dinámica y comprehensiva.

INDEX

Mots-clés : assimilation, concept, identité collective, recherche, relations interculturelles, sociologie, ville

\section{AUTEUR}

PIERRE TRIPIER

Professeur émérite de Sociologie, Laboratoire Printemps, Université de Versailles-Saint Quentinen-Yvelines, 47-49 Boulevard Vauban, 78045 Guyancourt Cedex. 